\title{
Radiodensidade hepática de cães hígidos por tomografia computadorizada helicoidal
}

\author{
Hepatic radiodensity in healthy dogs by helical computed tomography
}

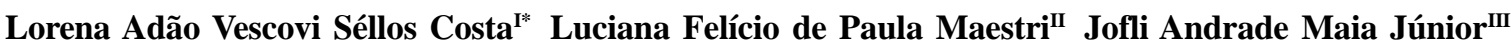 \\ Vicente Colombi Silva ${ }^{\mathrm{I}}$ Adrienne Brêtas Lanis ${ }^{\mathrm{I}}$ Bernardo Fernandes Lopes ${ }^{\mathrm{I}}$ Fabiano Séllos Costa
}

\section{RESUMO}

Os objetivos do presente estudo são obter valores médios de radiodensidade do parênquima hepático de cães normais e descrever a adaptação na espécie canina de uma técnica de mensuração da densidade tomográfica já utilizada em humanos. Para a realização do estudo, foram adquiridos exames tomográficos do fígado de oito cães hígidos adultos jovens, sem distinção de sexo ou raça. Após a realização do exame, foram obtidos valores médios de radiodensidade de três regiões de interesse (ROIs) do parênquima hepático em três diferentes níveis de corte. Além disso, foi realizada a análise de uma região de interesse do parênquima esplênico em três diferentes níveis de corte para uma avaliação comparativa entre os órgãos. Observou-se, no grupo avaliado, um valor

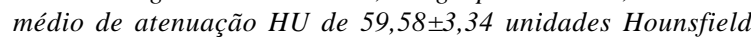
(HU). A diferença de densidade entre o parênquima hepático

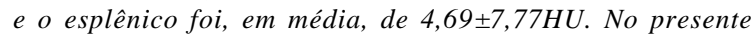
estudo, o exame de tomografia computadorizada demonstrou ser uma técnica com alta sensibilidade para estimar a radiodensidade do parênquima hepático. O estabelecimento de valores de normalidade e a padronização de uma metodologia de avaliação permitem melhor caracterização de alterações na radiodensidade pelo exame tomográfico, auxiliando no estabelecimento de um diagnóstico $e$ minimizando a necessidade de exames invasivos.

Palavras-chave: TC, fígado, radiodensidade hepática, canino.

\section{ABSTRACT}

The objectives of the study reported here were to obtain medium values of hepatic parenchyma radiodensity in healthy dogs, and also describe the adaptation in canine specie of a tomographic density measurement technique, which has already been used in humans. For the accomplishment of this study tomographic exams were realized in eight young dogs without sex or breed definition. After the exams, the liver's degree of $\mathrm{HU}$ attenuation was determined through a mean value of three selected regions of interest (ROI) in hepatic parenchyma obtained in three different levels of cut. Additionally, a region of interest in the splenic parenchyma was analyzed in three different levels of cut for comparative evaluation between the organs. In the experimental group it

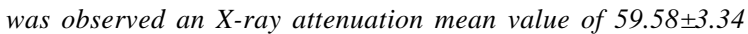
Hounsfield Units (HU). The density difference between hepatic and splenic parenchyma was a mean value of $4.69 \pm 7.77 \mathrm{HU}$. In the present study, it was observed that computed tomography is a technique with high sensitivity to estimate hepatic parenchyma density. The establishment of reference values and the standardizing of an evaluation methodology allow a better characterization of radiodensity changes in the tomographic exams, assisting in establishing a diagnosis and minimizing the necessity of invasive testing to the patient.

Key words: CT, liver, hepatic radiodensity, canine.

\section{INTRODUÇÃO}

O fígado é um órgão relativamente grande e corresponde a cerca de 3 a $4 \%$ do peso corporal dos cães, possuindo tamanho, formato, localização e radiopacidade bem determinados radiologicamente na maioria dos pacientes caninos (TIDWELL, 2007). Diversas alterações que ocorrem no fígado podem causar danos ao animal muitas vezes irreparáveis, por isso o diagnóstico precoce de inúmeras enfermidades é de fundamental importância para evitar maiores danos ao organismo (BUNCH, 2004).

\footnotetext{
IDepartamento de Medicina Veterinária, Centro de Ciências Agrárias (CCA), Universidade Federal do Espírito Santo (UFES). Alto Universitário, s/n, CP 16, 29500-000, Alegre, ES, Brasil. E-mail: lovescovi@gmail.com. *Autor para correspondência.

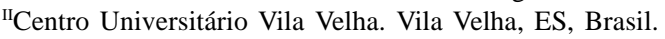

IIICentro de Escolas de Formação Especial Técnica em Radiologia e Imagenologia do Espírito Santo, Vila Velha, ES, Brasil.
} 
A tomografia computadorizada (TC) é um método radiológico que permite mensurar o grau de absorção da radiação- $X$ dos volumes elementares de um corte, possibilitando estimar a radiodensidade com precisão das estruturas avaliadas (DOYON et al., 2004). Em medicina veterinária, a utilização e os estudos da TC tiveram início em 1980 (FIKE, et al., 1980; SMALLWOOD \& GEORGE II, 1992a, 1992b, 1993). No entanto, apesar de a TC atualmente ser uma realidade dentro dos métodos diagnósticos em medicina veterinária, ainda apresenta limitação quanto suas aplicações (PROBST \& KNEISSL, 2001; OHLERTH \& SCHARF, 2007). Segundo FERREIRA et al. (1998), a TC é um método excelente para a avaliação das cavidades abdominal e pélvica, podendo fornecer mais detalhes referentes às alterações de radiodensidade que um exame de ultrassonografia ou de radiologia convencional.

Para se considerar qualquer método de diagnóstico por imagem eficiente, é imprescindível o conhecimento detalhado da apresentação normal de cada órgão, assim como das alterações espécieespecíficas em todos os processos patológicos e em todos os possíveis órgãos a serem examinados (SMALLWOOD \& GEORGE II, 1992a, 1992b, 1993; FERREIRA et al., 1998). Assim, a partir da caracterização das densidades normais dos tecidos, torna-se possível a determinação de focos de menor densidade, correspondendo, por exemplo, a áreas de necrose ou mesmo áreas de maior densidade tecidual como neoplasias (FERREIRA et al., 1998).

O fígado pode sofrer lesões focais ou mesmo difusas que podem alterar sua anatomia ou radiodensidade (DOYON et al., 2004). Segundo TIDWELL (2007), a TC possui a habilidade de auxiliar no diagnóstico de grande parte das enfermidades que acometem o organismo animal, uma vez que se manifestem nas imagens tomográficas formadas. De acordo com DOYON et al., (2004), o exame de TC sem a utilização de contraste iodado endovenoso permite mensurar a radiodensidade do parênquima hepático normal e na presença de eventuais alterações.

O grau de atenuação HU fornecido pela TC é considerado importante em humanos para o diagnóstico e monitoramento de pacientes com enfermidades que alteram a densidade do parênquima hepático, tais como esteatose e glicogenose (ROCKALL et al., 2003; KODAMA et al., 2007). Segundo KODAMA et al. (2007), em humanos, é de grande importância uma avaliação comparativa do grau de atenuação radiográfica do fígado com o baço para melhor esclarecimento do processo. A densidade do parênquima hepático é normalmente sempre superior à do parênquima esplênico (DOYON et al., 2004; KODAMA et al., 2007), e a diferença entre a atenuação HU desses órgãos constitui o critério diagnóstico mais utilizado em humanos, na prática clínica (SHIGUEOKA, 2001).

O objetivo do presente trabalho é estabelecer valores de normalidade da radiodensidade hepática em cães por tomografia computadorizada, utilizando como padrão a adaptação da técnica descrita por KODAMA et al. (2007).

\section{MATERIAL E MÉTODOS}

Para a realização desse experimento, foram utilizados oito cães adultos jovens, pesando entre 10 e $15 \mathrm{~kg}$, sem distinção sexual ou racial e sem alterações que pudessem interferir nos resultados da pesquisa. Todos os cães foram provenientes do Centro de Controle de Zoonoses do município de Vila Velha, Espírito Santo(ES), Brasil.

Previamente ao início da realização das análises tomográficas, os animais foram submetidos a testes de triagem, incluindo uma acurada avaliação clínica, ultrassonográfica e hematológica e realizandose a dosagem sérica dos níveis de albumina, de uréia e das enzimas ALT, AST, FA e GGT. Os animais que se apresentaram normais foram selecionados para o grupo experimental, sendo posteriormente vermifugados e vacinados, ficando 40 dias em observação e recebendo água ad libitum e ração padronizada (Purina ProPlan ${ }^{\circledR}$ adulto). Ao término das análises, todos os cães foram doados.

Anteriormente à realização dos exames, os cães foram submetidos a um jejum alimentar durante 12 horas. Todos os animais foram canulados com cateter de 22 ou 24 gauge na veia cefálica e submetidos a um protocolo de contenção química com administração intravenosa de diazepam $\left(0,5 \mathrm{mg} \mathrm{kg}^{-1}\right)$. Durante o procedimento, os animais receberam fluidoterapia com solução fisiológica em uma velocidade aproximada de infusão de $10 \mathrm{ml} \mathrm{kg}^{-1} \mathrm{~h}^{-1}$ ( $\mathrm{NaCl}$ 0,9\%), permitindo suporte da função cardiovascular e renal e servindo como via de acesso para administração dos fármacos utilizados. A anestesia geral foi induzida e mantida com propofol $\left(6 \mathrm{mg} \mathrm{kg}{ }^{-1}\right)$, por via intravenosa, sob dose e efeito.

Para a realização do exame, os animais foram mantidos em decúbito lateral direito sobre a mesa de exames. As imagens tomográficas foram adquiridas em tomógrafo helicoidal (GE Hi-Speed FXI), com 120kVp e auto-mA, na velocidade de uma rotação $\mathrm{s}^{-1}$. Foram realizados cortes transversais de $2 \mathrm{~mm}$ de espessura, obtidos desde a cúpula diafragmática até as cristas ilíacas, com intervalos entre cortes de $1 \mathrm{~mm}$. Todas as 
imagens foram realizadas com filtro de partes moles, e o aparelho foi devidamente calibrado antes da aquisição das imagens tomográficas.

Após o exame tomográfico e a digitalização das imagens, calculou-se o valor de atenuação em HU do fígado a partir da média de três regiões de interesse (ROIs - regions of interest) e uma região de interesse no baço, seguindo três níveis representativos. Os níveis para avaliação da radiodensidade hepática e esplênica foram realizados em três cortes diferentes dos órgãos em questão. Para o fígado, foram selecionados os ROIs no lobo lateral esquerdo, lobo lateral direito e lobo quadrado do fígado (Figura 1) e, para o baço, foi selecionada a medida na região de seu maior volume (Figura 2).

Cada ROI apresentava uma área de $25 \pm 0,1 \mathrm{~mm}^{2}$. Posteriormente à avaliação, foi estabelecida uma relação entre a densidade tomográfica do fígado e do baço. Para análise dos resultados, foi realizada estatística descritiva das variáveis, obtendo-se os valores médios e o respectivo desvio padrão.

\section{RESULTADOS E DISCUSSÃO}

A radiodensidade do parênquima hepático observada no grupo de estudo avaliado apresentou um valor médio de atenuação HU de 59,58ะ3,34HU nos três níveis realizados (Tabela 1 ). Avaliando-se comparativamente o baço e o fígado, verificou-se uma maior atenuação promovida pelo parênquima hepático. A diferença de densidade entre o parênquima hepático e o esplênico foi, em média, de 4,69₫7,77HU. Após a divisão dos valores médios da densidade do fígado pelo baço, a relação verificada entre a atenuação HU

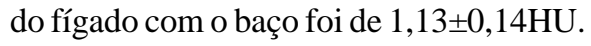

Apesar da sua importância para um estudo comparativo, poucos trabalhos na literatura consultada (FERREIRA, 1998; CÁCERES et al., 2006) fornecem informações sobre a radiodensidade do parênquima hepático de cães normais. Segundo OHLERTH \& SCHARF (2007), a TC apresenta uma grande sensibilidade a pequenas diferenças de atenuação dos raios-X, em comparação com os exames radiográficos convencionais. Dessa forma, é preciso considerar que a quantificação da radiodensidade pela técnica de TC fornece informações com alta precisão, em comparação com outros métodos de diagnóstico por imagem.

Em medicina veterinária, a TC é um método de imagem atual e em plena expansão. Essa técnica veio para incrementar ainda mais o diagnóstico de doenças, fornecendo informações valiosas e complementares às obtidas pelos métodos de

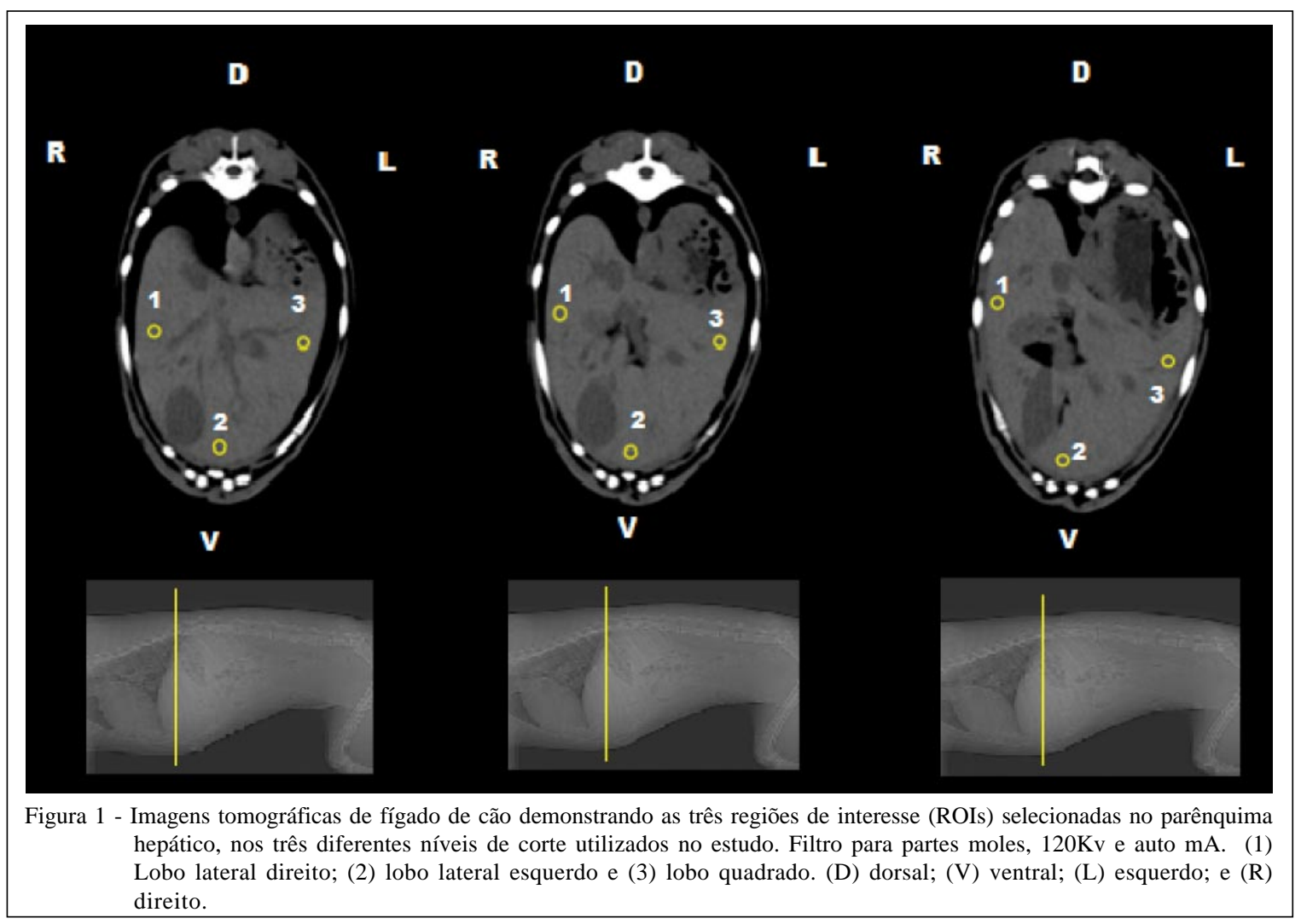

Ciência Rural, v.40, n.4, abr, 2010. 


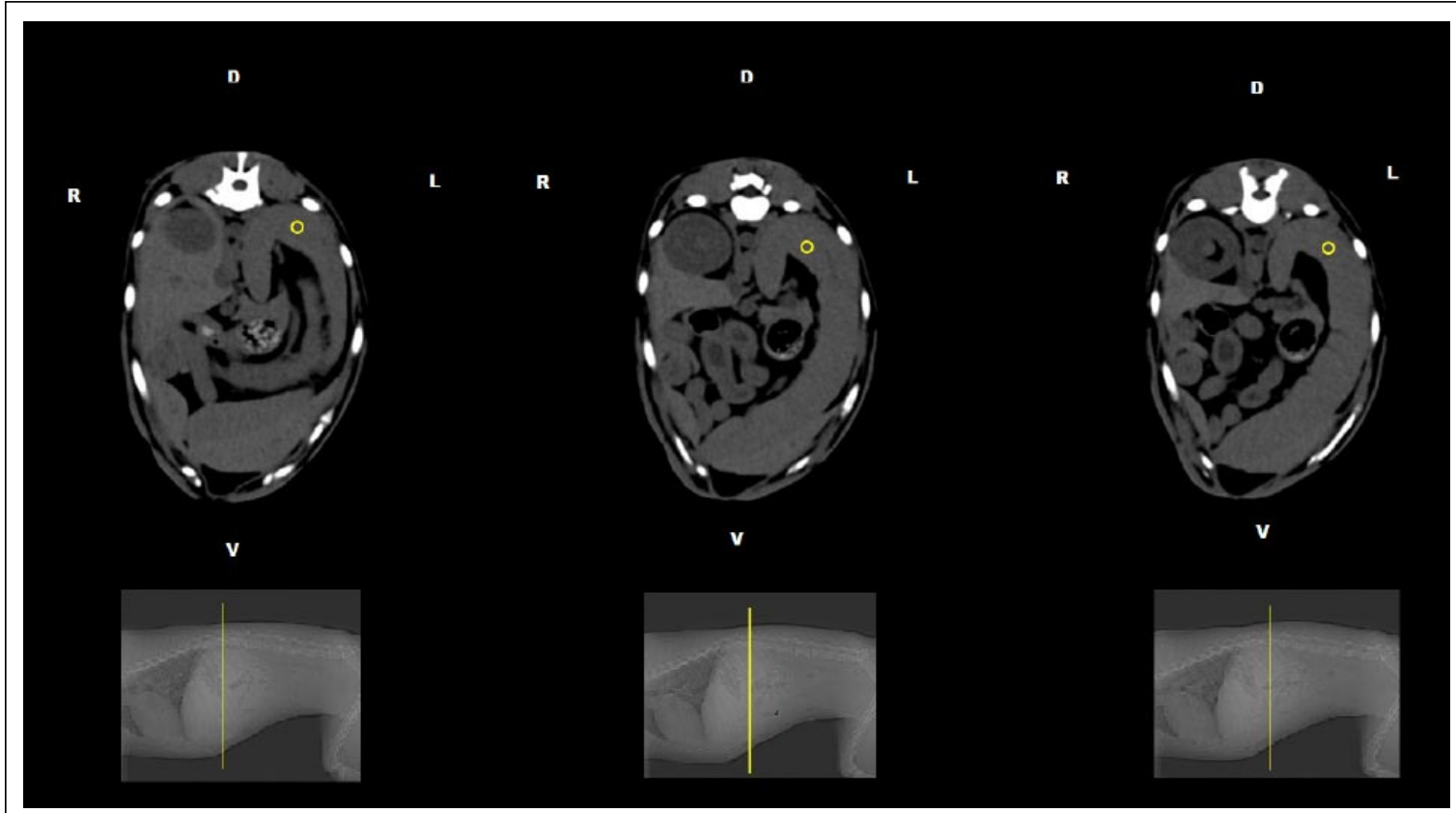

Figura 2 - Imagens tomográficas do baço de cão demonstrando a região de interesse (ROI) selecionada no parênquima esplênico, nos três níveis de corte utilizados no estudo. Filtro para partes moles, $120 \mathrm{Kv}$ e auto mA. (D) dorsal; (V) ventral; (L) esquerdo; e (R) direito.

diagnóstico por imagem convencionais, como os exames radiográficos e ultrassonográficos. Diversas alterações vasculares e parenquimatosas podem ser diagnosticadas por meio da TC, em que a utilização de contraste iodado auxiliará na interpretação do exame tomográfico, sendo possíveis caracterizações numéricas precisas e localização de lesões hepáticas em toda extensão do órgão (WINTER et al., 2005; OHLERTH \& SCHARF, 2007).

Para a determinação da radiodensidade do parênquima do fígado, não foi realizada a utilização de contraste intravenoso no grupo experimental, no momento do exame. Segundo DOYON et al. (2004), apesar do favorecimento da identificação das estruturas vasculares, o contraste radiográfico interfere diretamente na sua radiodensidade, fornecendo um resultado irreal.

Uma das maiores vantagens da TC para medicina veterinária, segundo WINTER et al. (2005) e TEIXEIRA et al. (2007), é a rapidez na realização do exame, o que reduz os artefatos de movimento e possibilita que os animais fiquem pouco tempo anestesiados, sendo um método não invasivo e seguro. Esses aspectos também foram observados na realização dos exames tomográficos da presente pesquisa.

Assim como em outros estudos (SMALLWOOD \& GEORGE, 1993; CÁCERES et al., 2006; TEIXEIRA et al. 2007), os exames de triagem previamente realizados em cada animal do grupo experimental permitiram selecionar um grupo de animais sem alterações clínicas, laboratoriais e ultrassonográficas indicativas de alguma hepatopatia que pudesse alterar a anatomia do órgão, sua radiodensidade, ou ambos.

A metodologia aplicada neste estudo para a mensuração da radiodensidade do parênquima hepático foi uma adaptação da metodologia utilizada em medicina humana descrita por KODAMA et al. (2007). Nessa metodologia, o fígado é dividido em diferentes seções, definidas por um método chamado de Sistema de Couinaud, permitindo, assim, a escolha da área exata de mensuração a partir das regiões de interesse, fazendo com que haja padronização dos valores obtidos. Essa metodologia foi facilmente adaptada para a avaliação tomográfica do fígado de cães, possibilitando, assim, como em humanos, uniformizar o ponto de análise da radiodensidade do parênquima hepático.

Assim como citado em humanos (SHIGUEOKA, 2001; DOYON et al., 2004; KODAMA et al., 2007), é preciso considerar a relação da densidade do fígado e do baço para a melhor interpretação das hepatopatias difusas que acometem os cães. No presente estudo, os resultados demonstraram que o fígado apresentou valores médios de radiodensidade maiores que o baço. 
Tabela 1 - Medidas da média e do desvio padrão da densidade hepática em Unidades Hounsfield (HU), nos três níveis de interesse por tomografia computadorizada, em cães hígidos. Alegre - ES, 2009.

\begin{tabular}{|c|c|c|c|c|}
\hline \multirow{2}{*}{ Animal } & \multicolumn{3}{|c|}{ Média de densidade no nível Média de densidade no nível Média de densidade no nível } & \multirow{2}{*}{$\begin{array}{l}\text { Média total dos } \\
\text { níveis/animal }\end{array}$} \\
\hline & 1 & 2 & 3 & \\
\hline 1 & 56,73 & 66,67 & 66,00 & 63,13 \\
\hline 2 & 60,98 & 67,00 & 64,33 & 64,10 \\
\hline 3 & 61,91 & 61,33 & 55,33 & 59,53 \\
\hline 4 & 57,76 & 52,66 & 57,67 & 56,03 \\
\hline 5 & 64,77 & 60,00 & 57,33 & 60,70 \\
\hline 6 & 64,27 & 57,33 & 60,33 & 60,65 \\
\hline 7 & 53,02 & 62,67 & 59,00 & 58,23 \\
\hline 8 & 57,09 & 49,67 & 56,00 & 54,25 \\
\hline Média total & 59,57 & 59,67 & 59,50 & 59,58 \\
\hline Desvio padrão & 4,73 & 6,20 & 3,86 & 3,34 \\
\hline
\end{tabular}

A pesquisa realizada por KODAMA et al. (2007) permitiu que os níveis escolhidos para as mensurações da atenuação HU fossem comuns entre o fígado e o baço, uma vez que a anatomia humana permite a localização dos dois órgãos no mesmo plano de corte. Em razão de a anatomia canina não permitir, na maioria dos casos, a identificação do fígado e baço no mesmo plano de corte, foram escolhidos três níveis hepáticos representativos e similares para todos os cães avaliados e três diferentes níveis para a análise esplênica.

Na presente pesquisa, foi padronizada a área das nove regiões de interesse estudadas no fígado e das três estudadas no baço de cada cão, sendo posteriormente calculada a estatística descritiva das variáveis obtidas. Segundo KODAMA et al. (2007), a padronização dos tamanhos dos ROIs é importante e fornece maior precisão aos resultados.

O valor médio encontrado dos três níveis avaliados da radiodensidade hepática foi de $59,58 \pm 3,34 \mathrm{HU}$, sendo próximo ao valor encontrado nos estudos de CÁCERES et al. (2006), em que a média do valor de atenuação HU por ele encontrado foi de 65 HU, oscilando entre 60 e 70HU, após a análise do fígado de cinco animais hígidos. No entanto, em um estudo realizado por FERREIRA (1998), o valor da média da radiodensidade hepática para cães normais foi de 81,31ะ6,98HU, um valor médio elevado, em comparação com os demais trabalhos, podendo estar relacionado à calibração do tomógrafo utilizado no experimento ou mesmo à espessura de corte utilizada. Vale ressaltar que a metodologia adotada pelos autores difere da utilizada no presente trabalho, não havendo citação sobre a padronização dos pontos de análise. Concordando com as afirmações de KODAMA et al. (2007), torna-se importante a descrição precisa da metodologia e da seleção dos pontos de análise, visando a aumentar a precisão das mensurações de radiodensidade hepática para evitar equívocos na interpretação dos achados tomográficos.

Algumas alterações difusas que acometem o fígado e alteram sua densidade podem não ser diferenciadas clinicamente ou por outros métodos de diagnóstico por imagem. Exemplo disso é o acúmulo de glicogênio hepático que promove aumento na radiodensidade e esteatose hepática, promovendo diminuição(OHLERTH \& SCHARF, 2007). Dessa forma, a padronização de valores de normalidade e a caracterização de alterações na atenuação da radiação$\mathrm{X}$ pelo exame tomográfico podem auxiliar no estabelecimento de um diagnóstico e minimizar a necessidade de exames invasivos ao paciente.

\section{CONCLUSÕES}

Confirma-se que a TC é uma técnica de diagnóstico por imagem que possibilitou a quantificação da densidade do parênquima em Unidades Hounsfield. Ressalta-se a importância desse método de exame complementar na medicina veterinária, visando a diagnosticar precocemente e de forma mais precisa as alterações que acometem o fígado de cães. Os resultados médios de radiodensidade hepática utilizando a metodologia de segmentação anatômica pelo sistema de Couinaud em cães demonstraram valores inferiores aos descritos na literatura, para essa espécie.

\section{COMITÊ DE ÉTICA E BIOSSEGURANÇA}

Este estudo foi realizado sob aprovação do Comitê de Ética e Bem-estar Animal da Universidade Federal do Espírito Santo, tendo sido respeitados todos os preceitos éticos de proteção aos animais.

Ciência Rural, v.40, n.4, abr, 2010. 


\section{REFERÊNCIAS}

BUNCH, S.E. Distúrbios hepáticos agudos e sistêmicos que acometem o fígado. In: ETTINGER, S.J.; FELDMAN, E.C. Tratado de medicina interna veterinária: doenças do cão e do gato. 5.ed. Rio de Janeiro : Guanabara Koogan, 2004. p.1398-1412.

CÁCERES, A.V. et al. Helical computed tomographic angiography of the normal canine pancreas. Radiology \& Ultrasound, v.47, n.3, p.270-278, 2006. Disponível em: $<\mathrm{h} \mathrm{t} \mathrm{t} \mathrm{p} \mathrm{:} \mathrm{/} \mathrm{/} \mathrm{a} \mathrm{p} \mathrm{p} \mathrm{s.i} \mathrm{s} \mathrm{i} \mathrm{k} \mathrm{n} \mathrm{o} \mathrm{w} \mathrm{l} \mathrm{e} \mathrm{d} \mathrm{g} \mathrm{e.} \mathrm{c} \mathrm{o} \mathrm{m} \mathrm{/}$ full_record.do?product=UA\&search_mode=Generalsea $\mathrm{r}$ c h \& q i d $=1 \& \mathrm{~S} \mathrm{I} \mathrm{D}=1 \mathrm{C}$ i 93 b 8 B 8 G PjLf3NMFg\&page $=1 \&$ doc $=1 \&$ colname $=$ WOS $>$. Acesso em: 11 jan.2010. doi: 10.1111/j.1740-8261.2006.00139.x.

DYCE, K.M. et al. Tratado de anatomia veterinária. 3.ed. Rio de Janeiro: Saunders Elsevier, 2004. 663p.

DOYON, D. et al. Tomografia computadorizada. 2.ed. Rio de Janeiro: MEDSI, 2004. 392p.

FERREIRA, F.M. Avaliação estrutural do parênquima hepático através da histologia e tomografia computadorizada em cães intoxicados experimentalmente pelo tetracloreto de carbono. 1998. 83f. Tese (Doutorado em Medicina Veterinária) - Universidade Federal do Paraná, Curitiba, PR.

FERREIRA, F.M. et al. A tomografia computadorizada em medicina veterinária: Uma breve descrição do método e suas aplicações clínicas. Revista Clínica Veterinária, v.3, n.12, p.27-32, 1998.

FIKE, J.R. et al. Canine anatomy as assessed by computerized tomography. American Journal Veterinary Research, v.41, p.1823-1832, 1980. Disponível em: <http://apps.isiknowledge.com/ f u l l_ re c ord.d o ? p r o d u c t $=$ U A \& s e a r c h $\operatorname{mode}=\mathrm{G}$ en er a l S e a r c h \& q id $=6$ \& S I D $=1 \mathrm{C}$ i $93 \mathrm{~b}$ 8B8GPjLf3NMFg\&page $=1 \&$ doc $=1 \&$ colname $=$ WOS $>$. Acesso em: 11 jan. 2010.

KODAMA, Y. et al. Comparison of CT methods for determining the fat content of the liver. American Journal of Roentgenology, v.188, p.1307-1321, 2007. Disponível em: <http://www.ajronline.org/cgi/content/full/188/5/1307>. Acesso em: 11 jan. 2010. doi:. 10.2214/AJR.06.0992.

OHLERTH, S.; SHARF, G. Computed tomography in small animals: Basic principles and state of the art applications. Veterinary Journal, v.173, p.254-271, 2007. Disponível em: <http:// www.sciencedirect.com/science?_ob=ArticleURL\&_udi=B6WXN4JD0HC9-1\&_user $=7088798 \&$ \&doc $=1 \&$ \& fmt $=$ \&_orig $=$ searc h \&_sort $=$ d \&_docanchor $=\&$ view $=$ c $\&$ _searchStrId $=1162328695 \&$ \&erunOrigin $=$ google $\&$ _acct $=$ C 00005 $0221 \&$ _ version $=1 \&$ \&_urlversion $=0 \&$ \&_useri d $=7$ 088798\&md5=65cbd2e79afce7c8447e80d65eee2638>. Acesso em: 11 jan. 2010. doi: 10.1016/j.tvjl.2005.12.014.

PROBST, A.; KNEISSL, S. Computed tomographic anatomy of the canine pancreas. Veterinary Radiology \& Ultrasound v.42, n.3, p.226-230, 2001. Disponível em: <http://
apps.isiknowledge.com/full_record.do?product=UA\&search_ $\mathrm{mod}$ e $=\mathrm{G}$ e n e r a l S e a r c h \& q i d $=8$ \& S I D $=\overline{1}$ Ci93b8B8GPjLf3NMFg\&page $=1 \&$ doc $=2 \&$ colname $=$ WOS $>$. Acesso em: 11 jan. 2010.

ROCKALL, A.G. et al. Hepatic steatosis in Cushing’s syndrome: a radiological assessment using computed tomography. European Journal of Endocrinology, v.149, p.543-548, 2003. Disponível em: <http://www.eje-online.org/cgi/reprint/ 149/6/543.pdf>. Acesso em: 11 jan.2010.

SHIQUEOKA, D.C. Avaliação quantitativa da esteatose hepática pela tomografia computadorizada. 2001. 60f. Tese (Doutorado em Diagnóstico por Imagem) - Universidade Federal de São Paulo, SP.

SMALLWOOD, J.E.; GEORGE II, T.F. Anatomic atlas for computed tomography in the mesaticephalic dog: head and neck. Veterinary Radiology \& Ultrasound, v.33, n.4, p.217-240, 1992a. Disponível em: <http://apps.isiknowledge.com/full_record. do ? product $=$ UA \& s e arch_mode=Generalsea r c h \& q i d $=12$ \& S I D = 1 C i 93 b 8 B 8 G P j L f 3 NMFg\&page $=1 \&$ doc $=5 \&$ colname $=$ WOS $>$. Acesso em: 11 jan. 2010 .

SMALLWOOD, J.E.; GEORGE II, T.F. Anatomic atlas for computed tomography in the mesaticephalic dog: Caudal abdomen and pelvis. Veterinary Radiology \& Ultrasound, v.33, n.6, p.143-167, 1992b. Disponível em: <http://apps.isiknowledge.com/ full record.do? product $=$ UA \& search mode $=$ G e n e ra l S e a r c h \& q i d = 12 \& S I D $=1$ C i 93 b 8B8GPjLf3NMFg\&page $=1 \&$ doc $=3 \&$ colname $=$ WOS $>$. Acesso em: 11 jan. 2010.

SMALLWOOD, J.E.; GEORGE II, T.F. Anatomic atlas for computed tomography in the mesaticephalic dog: thorax and cranial abdomen. Veterinary Radiology \& Ultrasound, v.34, n.2, p.65-84, 1993. Disponível em: <http://apps.isiknowledge.com/ full record.do? product $=$ UA \& s e a r c h mode $=\mathrm{G}$ en eral S e arch \& qid $=12 \& \mathrm{SID}=1 \mathrm{C}$ i 93 b 8 B $8 \mathrm{G}$ PjLf3NMFg\&page $=1 \&$ doc $=4 \&$ colname $=$ WOS $>$. Acesso em: 11 jan. 2010.

TEIXEIRA, M. et al. Helical computed tomographic anatomy of the canine abdomen. Veterinary Journal, v.174, p.133138, 2007. Disponível em: <http://apps.isiknowledge.com/ f u l l_ record.do ? p roduct $=$ U A \& s e a r c h_m o $\mathrm{de}=\mathrm{GeneralSe}$ arch \& qid $=17 \& \mathrm{SID}=1 \mathrm{Ci} 93 \mathrm{~b} 8 \mathrm{~B} 8 \mathrm{GPjL}$ $\mathrm{f} 3 \mathrm{NMFg} \&$ page $=1 \&$ doc $=1 \&$ colname $=$ WOS $>$. Acesso em: 11 jan. 2010. doi: 10.1016/j.tvjl.2006.04.005.

TIDWELL, A.S. Principle of computed tomography and magnetic resonance imaging. In: THRALL, D.E. Veterinary Diagnostic Radiology. 5.ed. Philadelphia: Saunders Elsevier, 2007. Cap.4, p.50-77.

WINTER, M.D. et al. Three-dimensional helical computed tomographic angiography of the liver in five dogs. Veterinary Radiology \& Ultrasound, v.46, n.6, p.494-499, 2005. Disponível em: <http:// apps.isiknowledge.com/full_record.do?product=UA\&search_mode $=\mathrm{G}$ e n e r a l S e a r c h \& q i d $=19 \& \mathrm{~S} \mathrm{I} \mathrm{D}=1 \mathrm{C}$ i93b8B8GPjLf3NMFg\&page $=1 \&$ doc $=1 \&$ colname $=$ WOS $>$. Acesso em: 11 jan. 2010. doi: 10.1111/j.1740-8261.2005.00090zx. 